\section{Atividade física e \\ comportamento sedentário em adolescentes estudantes do ensino médio}

\section{Physical activity and sedentary behavior among adolescent high school students}

\section{Maria Cecília Marinho Tenório',"II \\ Mauro Virgilio Gomes de Barros ${ }^{1,11}$ \\ Rafael Miranda Tassitano ${ }^{1,1,1,11}$ \\ Jorge Bezerra ${ }^{1,11}$ \\ Juliana Marinho Tenório" \\ Pedro Curi Hallal'v}

' Universidade de Pernambuco, Programa de Pós-graduação em Hebiatria, Programa Associado de Pós-graduação em Educação Física.

"Grupo de Pesquisa em Estilos de Vida e Saúde. Recife, PE, Brasil.

"'Universidade Federal Rural de Pernambuco.

IVUniversidade Federal de Pelotas, Programa de Pós-graduação em Educação Física, Programa de Pós-Graduação em Epidemiologia.

Fonte financiadora: Conselho Nacional de Desenvolvimento Científico e Tecnológico - CNPq Processo - 486023/2006-0

Correspondência: Maria Cecília Marinho Tenório. Rua Pereira de Moraes, 426, Cordeiro, Recife, PE CEP 50.630-610. E-mail: mariaceciliatenorio@gmail.com

\section{Resumo}

Objetivo: Identificar a prevalência e fatores associados a nível insuficiente de prática de atividades físicas e exposição a comportamento sedentário em adolescentes. Métodos: Estudo transversal cuja amostra foi constituída por 4210 estudantes do ensino médio (14-19 anos), selecionados através de amostragem por conglomerados em dois estágios. Para coleta de dados foi utilizado o questionário "Global School-based Student Health Survey". Sujeitos que relataram participar de, pelo menos, 60 minutos diários de atividades físicas moderadas a vigorosas, durante cinco ou mais dias por semana, foram classificados como ativos, enquanto os demais foram denominados insuficientemente ativos. Foram considerados expostos a comportamento sedentário aqueles que referiram assistir televisão por três horas ou mais por dia. As análises foram realizadas através do teste de Qui-quadrado e de regressão logística binária. Resultados: A prevalência de insuficientemente ativos foi $65,1 \%$ (IC95\% 63,7-66,6). Identificou-se que sexo, local de residência (rural/urbano), turno de aulas, status ocupacional e participação nas aulas de educação física foram fatores associados a nível insuficiente de atividade física. Verificou-se prevalência de exposição a comportamento sedentário de 40,9\% (IC95\% 39,4-42,4) em dias de semana e de $49,9 \%$ (IC95\% 48,4-51,4) em dias de final de semana. Local de residência, turno de aulas, status ocupacional e participação nas aulas de educação física foram fatores associados à exposição a comportamento sedentário em dias de semana; enquanto sexo, faixa etária e turno de aulas discriminaram a exposição nos dias de final de semana. Conclusão: Este estudo evidenciou elevada prevalência de níveis insuficientes de atividade física e de exposição a comportamento sedentário.

Palavras-chave: Atividade motora. Adolescente. Epidemiologia. Conduta de saúde. 


\section{Abstract}

Objective: To identify the prevalence of factors associated with insufficient physical activity levels and sedentary behavior among adolescents. Methods: In this crosssectional study 4,210 students (14-19 years old) attending public high-schools were selected using random two-stage cluster sampling. Data were collected by using the Global School-based Student Health Survey. Subjects who reported performing 60 minutes or more of moderate to vigorous physical activity five days a week or more were classified as physically active, while the remainder was classified as insufficiently active. Subjects who reported watching TV three or more hours a day were considered to be exposed to sedentary behavior. Analyses were carried out by using Chi-square tests and binary logistic regression. Results: The prevalence of insufficiently active subjects was $65.1 \%$ (CI95\% 63.7-66.6). Gender, place of residence (rural/urban), time of day attending school, occupational status, and enrollment in physical education were identified as factors significantly associated with exposure to insufficient levels of physical activity. Prevalence of exposure to sedentary behavior was $40.9 \%$ (CI95\% 39.4-42.4) on week days and $49.9 \%$ (CI95\% 48.4-51.4) on weekend days. Place of residence, time of day attending school, occupational status and enrollment in physical education were identified as factors significantly associated with exposure to sedentary behavior on week days, whereas on weekend days exposure to sedentary behavior was related to gender, age and time of day attending school. Conclusion: This study showed a high prevalence of insufficient physical activity levels and exposure to sedentary behaviors among adolescents.

Keywords: Physical activity. Motor activity. Adolescent. Epidemiology. Health behavior.

\section{Introdução}

Os benefícios para a saúde e bem-estar decorrentes da prática regular de atividade física estão bem documentados ${ }^{1,2}$. Existe uma relação inversa entre o nível de atividade física e o risco de ocorrência de diversas doenças crônicas não transmissíveis, como o câncer ${ }^{3,4}$ e a hipertensão ${ }^{5,6}$. Além disso, as atividades físicas têm um papel fundamental na prevenção e controle da obesidade ${ }^{7,8}$.

Durante a adolescência, especificamente, há evidências de que a atividade física traz benefícios associados à saúde esquelética (conteúdo mineral e densidade óssea) e ao controle da pressão sangüínea e da obesidade ${ }^{2,9-11}$. Alguns estudos demonstraram, também, que programas de atividades físicas de intensidade moderada a vigorosa ajudam na redução da gordura visceral e dos níveis de triglicérides em crianças e adolescentes com sobrepeso ${ }^{12-14}$. Outros benefícios da prática regular da atividade física foram sintetizados em uma recente revisão sistamática ${ }^{15}$. Além disso, há evidências de que a prática de atividade física na adolescência pode estar associada ao nível de atividade física na vida adulta ${ }^{16}$.

Como conseqüência dessas evidências, instituições científicas de todo o mundo vêm trabalhando para definir a dose mínima de atividades físicas necessárias para derivar benefícios à saúde de adolescentes ${ }^{17-19}$. A mais recente, publicada em 2001, sugere que os jovens devem participar diariamente de atividades físicas de intensidade moderada a vigorosa por, pelo menos, 60 minutos ${ }^{20}$.

Apesar do reconhecimento da importância da atividade física como fator de promoção da saúde e de prevenção de doenças, a prevalência de exposição a baixos níveis de atividade física é elevada e parece afetar pessoas de todas as idades. No Brasil, de acordo com a região onde o levantamento foi realizado, o instrumento de medida utilizado e o contexto de vida ao qual as medidas se referem (lazer, deslocamentos, tarefas domésticas), os dados disponíveis sugerem que de $39 \%$ a 93\% dos adolescentes estão expostos a baixos níveis de atividade física ${ }^{21-25}$. 
Duas revisões sistemáticas sobre estudos epidemiológicos relacionados ao padrão de atividades físicas evidenciaram que, apesar do crescimento do número de estudos na área, poucos focalizaram sujeitos na adolescência ${ }^{15,26}$. Além disso, atualmente, há necessidade de avaliar tanto a exposição a baixo nível de atividade física quanto a comportamentos sedentários. Isto é importante porque existem evidências sugerindo que a inatividade física e o sedentarismo são comportamentos independentes e têm diferentes efeitos sobre a saúde ${ }^{27-31}$. .

Diante do exposto e considerando a relativa escassez de estudos que analisem simultaneamente tanto o padrão de atividades físicas quanto a exposição a comportamentos sedentários, pretende-se identificar a prevalência e fatores associados a nível insuficiente de prática de atividades físicas e exposição a comportamento sedentário em adolescentes.

\section{Métodos}

O presente estudo foi conduzido como parte de um projeto denominado "Estilos de Vida e Comportamento de Risco à Saúde de Estudantes do Ensino Médio de Pernambuco", desenvolvido pelo Grupo de Pesquisa em Estilos de Vida e Saúde da Universidade de Pernambuco. O estudo foi aprovado pelo Comitê de Ética em Pesquisa com seres humanos do Hospital Agamenon Magalhães, situado na cidade de Recife (Pernambuco).

A população alvo foi limitada aos estudantes da rede pública estadual de ensino médio do Estado de Pernambuco. Considerando-se todas as dependências administrativas (federal, estadual, municipal e privada), os sujeitos matriculados na rede pública estadual representavam na época cerca de $80 \%$ do total de estudantes do ensino médio em Pernambuco. $\mathrm{O}$ dimensionamento da amostra foi efetuado de forma a atender os diversos objetivos do projeto, que incluíam a avaliação da exposição a dez fatores comportamentais relacionados à saúde, dentre os quais a prática de atividades físicas e a exposição a comportamentos sedentários.

Para o cálculo do tamanho da amostra foram utilizados os seguintes critérios: população estimada em 353 mil sujeitos; intervalo de confiança de $95 \%$; erro amostral de 3 pontos percentuais; prevalência estimada em $50 \%$; e efeito de delineamento amostral estabelecido em quatro vezes o tamanho mínimo da amostra. Isto representaria uma amostra mínima de 4.217 sujeitos.

Procurou-se garantir que a amostra selecionada representasse a população alvo quanto à distribuição conforme região geográfica, em escolas de pequeno (menos de 200 alunos), médio (200 a 499 alunos) e grande porte (500 alunos ou mais), e nos períodos diurno e noturno. Alunos matriculados no período da manhã e da tarde foram agrupados numa única categoria (estudantes do período diurno). A distribuição regional foi observada pelo número de escolas existentes em cada uma das 17 GEREs (Gerências Regionais de Ensino).

Para seleção da amostra requerida, recorreu-se a um procedimento de amostragem aleatória estratificada em dois estágios, sendo que a "escola" e a "turma" representaram, respectivamente, as unidades amostrais no primeiro e no segundo estágio. Todas as escolas da rede pública estadual em Pernambuco foram consideradas elegíveis para inclusão no estudo. No primeiro estágio, adotou-se como critério de estratificação para realização do sorteio a densidade de escolas em cada microrregião do Estado (GEREs) por porte. No segundo estágio, considerou-se a densidade de turmas nas escolas sorteadas por período (diurno e noturno) como critério para sorteio das turmas nas quais os questionários seriam aplicados. Todos os estudantes das turmas sorteadas foram convidados a participar do estudo. Os sorteios foram realizados mediante geração de números aleatórios através do programa EpiInfo (versão 6).

A coleta dos dados foi realizada no período de abril a outubro de 2006. A aplicação do questionário foi efetuada por seis mestrandos do programa de pós-graduação em 
Hebiatria da Universidade de Pernambuco, que participaram de uma capacitação prévia para padronização dos procedimentos de coleta de dados. A aplicação dos questionários foi efetuada em sala de aula, sem a presença dos professores, para todos os alunos presentes, independente da idade dos mesmos.

A participação dos sujeitos foi voluntária, os questionários não continham qualquer tipo de identificação pessoal (nome, matrícula) e todos foram informados de que poderiam desistir em qualquer etapa da fase de coleta de dados. Os alunos foram continuamente assistidos pelos aplicadores (sempre dois por turma) para que pudessem esclarecer dúvidas e auxiliar o preenchimento das informações. Um termo de consentimento negativo (parental passive consent form) foi usado para obter dos pais de estudantes com idade inferior a 18 anos permissão para que os mesmos participassem do estudo. O termo continha indicação clara do objetivo do estudo e solicitava que os pais comunicassem à escola caso eles concordassem com a participação do(s) filho(s). Estudantes com 18 ou mais anos de idade assinavam o próprio termo, indicando a sua concordância em participar do estudo.

O questionário utilizado foi uma versão traduzida e previamente testada do Global School-based Student Health Survey, proposto pela Organização Mundial da Saúde (OMS), e que se encontra disponível para consulta no seguinte endereço eletrônico: www.who.int/chp/gshs/en. Os indicadores de validade concorrente e reprodutibilidade foram determinados em estudo piloto que antecedeu o planejamento metodológico do estudo sob análise. Indicadores de reprodutibilidade (consistência de medidas teste-reteste) foram de moderados a altos na maioria dos itens do instrumento, sendo que os coeficientes de concordância (índice kappa) variaram de 0,52 a 1,00.

As variáveis dependentes do estudo foram a exposição a nível insuficiente de prática de atividades físicas e a comportamento sedentário. Considerando-se as recomendações para a saúde ${ }^{20}$, todos os sujeitos que relataram praticar 60 minutos ou mais de atividades físicas de intensidade moderada a vigorosa em pelo menos cinco dias da semana foram classificados como "ativos", enquanto os demais foram considerados como expostos a nível insuficiente de prática de atividade física (insuficientemente ativos). A exposição a comportamento sedentário foi determinada pelo tempo em horas despendido assistindo televisão. Sujeitos que relataram assistir habitualmente três ou mais horas de televisão por dia foram considerados expostos a comportamento sedentário. Duas variáveis relativas à exposição a comportamento sedentário foram consideradas nas análises, uma refletindo a conduta do adolescente nos dias da semana e outra refletindo os finais de semana.

As variáveis independentes consideradas para todas as análises inferenciais foram gênero (masculino/feminino), turno das aulas (diurno/noturno), série escolar, faixa etária (14 a 16 anos/17 a 19 anos), cor da pele (branco/não branco), situação ocupacional (trabalha/não trabalha), morar com os pais, local de residência (urbana/rural), escolaridade materna ( $\leq 8$ anos/ $>8$ anos) e participação nas aulas de educação física (participa/não participa).

O procedimento de tabulação final dos dados foi efetuado através do programa EpiData, recorrendo-se à entrada dupla e, posteriormente, à comparação dos arquivos de dados gerados a fim de detectar e corrigir erros. Procedimentos eletrônicos de controle de entrada de dados também foram adotados mediante utilização de controles (limites) para os valores que poderiam ser digitados em cada campo.

A análise foi realizada através do programa SPSS para Windows (versão 11). Nas análises bivariáveis foram empregados os testes de Qui-quadrado e Qui-quadrado para tendência. Nas análises multivariáveis utilizou-se regressão logística binária separadamente para cada desfecho em estudo (nível insuficiente de prática de atividade física, exposição a comportamento 
sedentário em dias da semana e exposição a comportamento sedentário nos finais de semana). A abordagem hierárquica utilizada conforme a recomendação da literatura ${ }^{32}$ considerou três níveis: a) distal, no qual foram incluídas as variáveis demográficas (sexo, idade e cor da pele); b) intermediário, no qual foram incluídos os fatores socioeconômicos (local de residência, situação ocupacional, morar com os pais e escolaridade materna); e c) proximal, no qual foram incluídos fatores relacionados à escola (turno, série escolar e participação nas aulas de Educação Física). Todas as variáveis foram para a análise ajustada, independente do valor $p$ na análise bruta.

Na análise ajustada foi adotada abordagem hierárquica, recorrendo-se inicialmente ao ajustamento das variáveis do bloco distal para os demais fatores do mesmo nível. Em seguida, as variáveis do bloco intermediário foram controladas para as variáveis do mesmo nível e para aquelas do nível distal que permaneceram no modelo. Finalmente, na análise das variáveis do bloco proximal, recorreu-se ao ajustamento para os demais fatores do mesmo nível hierárquico e para aqueles dos níveis intermediário e distal que permaneceram no modelo. Adotou-se como critério para permanência do fator nas análises de regressão um valor $p$ inferior a 0,20 . Ao final, considerou-se significativamente associado aos desfechos sob análise aqueles cujo valor $p$ foi inferior a 0,05 .

\section{Resultados}

Foram visitadas 76 escolas $(11 \%$ do total de escolas estaduais do estado) em 44 municípios, o que representa $23 \%$ do total de municípios pernambucanos. Foram entrevistados 4.210 estudantes (14-19 anos), sendo $59,8 \%$ do sexo feminino. Oitenta e três estudantes foram convidados a participar do estudo, mas se recusaram a participar ou devolveram o questionário em branco (1,9\% de recusas/perdas). A amostra final representa 99,8\% do inicialmente previsto, mas permite analisar a associação entre as variáveis com possibilidade de detectar como significativas razões de odds de 0,75 ou inferiores (nível de confiança de 95\% e poder de $80 \%$ ). As características demográficas e socioeconômicas estão apresentadas na Tabela 1.

A maioria dos estudantes (65,1\%; IC95\% 63,7-66,6) apresentou níveis insuficientes de atividade física, sendo que a proporção de sujeitos expostos a esta conduta de risco foi significativamente maior entre as moças $(70,2 \%$; IC95\% $68,3-71,9)$ do que entre os rapazes $(57,6 \%$; IC95\% 55,2-60,0). Os resultados relativos à exposição a níveis insuficientes de atividade física segundo variáveis demográficas e socioeconômicas estão apresentados na Tabela 2.

Em relação à exposição a comportamentos sedentários, verificou-se uma prevalência de 40,9\% (IC95\% 39,4-42,4) nos dias de semana e 49,9\% (IC95\% 48,4-51,4) nos finais de semana. A proporção de rapazes e moças expostos a comportamentos sedentários em dias de semana não foi significativamente diferente. Nos finais de semana, a proporção de expostos a comportamento sedentário foi significativamente maior entre os rapazes (54,0\%; IC95\% 51,6-56,4), em comparação ao observado entre as moças $(47,1 \%$; IC95\% 45,1-49,1). Os resultados estão na Tabela 3.

Análises de regressão logística orientadas por abordagem hierárquica permitiram observar que a exposição a níveis insuficientes de atividade física neste grupo de adolescentes está significativamente associada ao gênero, local de residência, turno das aulas, série, situação ocupacional e participação nas aulas de educação física (Tabela 4).

O modelo hierárquico de regressão também evidenciou que a faixa etária, o local de residência, o turno de aulas, o status ocupacional e a participação nas aulas de educação física foram identificados como fatores associados à exposição a comportamento sedentário nos dias de semana (Tabela 5). Observou-se, ainda, que sexo, faixa etária, situação ocupacional e turno de aulas discriminaram a exposição a esta conduta de risco à saúde nos finais de semana (Tabela 
Tabela 1 - Características demográficas e socioeconômicas dos sujeitos $(n=4210)$.

Table 1 - Demographic and socioeconomic characteristics of subjects $(n=4,210)$.

\begin{tabular}{|c|c|c|c|c|c|c|}
\hline \multirow[t]{2}{*}{ Variáveis } & \multicolumn{2}{|c|}{ Rapazes } & \multicolumn{2}{|c|}{ Moças } & \multicolumn{2}{|c|}{ Todos } \\
\hline & $\%$ & $\mathrm{~N}$ & $\%$ & $\mathrm{~N}$ & $\%$ & $\mathrm{~N}$ \\
\hline \multicolumn{7}{|l|}{ Faixa etária } \\
\hline $14-16$ & 35,5 & 599 & 46,4 & 1167 & 42,0 & 1176 \\
\hline $17-19$ & 64,5 & 1089 & 53,6 & 1346 & 58,0 & 2435 \\
\hline \multicolumn{7}{|l|}{ Cor da Pele } \\
\hline Branco & 24,8 & 417 & 25,5 & 639 & 25,2 & 1056 \\
\hline Não branco & 75,2 & 1263 & 74,5 & 1868 & 74,8 & 3131 \\
\hline \multicolumn{7}{|l|}{ Estado Civil } \\
\hline Solteiro & 95,8 & 1606 & 93,6 & 2340 & 94,4 & 3949 \\
\hline Outros & 4,2 & 71 & 6,4 & 161 & 5,6 & 234 \\
\hline \multicolumn{7}{|l|}{ Trabalho } \\
\hline Não & 69,3 & 1158 & 84,8 & 2121 & 78,6 & 3279 \\
\hline Sim & 30,7 & 514 & 15,2 & 381 & 21,4 & 895 \\
\hline \multicolumn{7}{|l|}{ Local de residência } \\
\hline Urbano & 78.1 & 1312 & 79.6 & 1985 & 79.0 & 3297 \\
\hline Rural & 21.9 & 367 & 20.4 & 510 & 21.0 & 877 \\
\hline \multicolumn{7}{|l|}{ Região } \\
\hline Metropolitana & 39,8 & 671 & 43,2 & 1085 & 41,8 & 1756 \\
\hline Zona da Mata & 18,1 & 306 & 17,3 & 434 & 17,6 & 740 \\
\hline Agreste & 16,5 & 279 & 16,8 & 423 & 16,7 & 702 \\
\hline Sertão & 14,9 & 251 & 12,9 & 323 & 13,7 & 574 \\
\hline Sertão São Francisco & 10,7 & 181 & 9,9 & 248 & 10,2 & 429 \\
\hline \multicolumn{7}{|c|}{ Escolaridade da mãe (anos de estudo) } \\
\hline$<8$ & 69,4 & 1087 & 74,5 & 1772 & 72,5 & 2859 \\
\hline$\geq 8$ & 30,6 & 479 & 25,5 & 607 & 27,5 & 1086 \\
\hline \multicolumn{7}{|l|}{ Turno } \\
\hline Diurno & 53,9 & 909 & 60,1 & 1508 & 57,6 & 2417 \\
\hline Noturno & 46,1 & 778 & 39,9 & 1002 & 42,4 & 1780 \\
\hline \multicolumn{7}{|l|}{ Série } \\
\hline $1^{\circ}$ ano & 46,2 & 779 & 43,8 & 1101 & 44,8 & 1880 \\
\hline $2^{\circ}$ ano & 30,9 & 522 & 32,6 & 818 & 31,9 & 1340 \\
\hline $3^{\circ}$ ano & 22,9 & 386 & 23,6 & 593 & 23,3 & 979 \\
\hline
\end{tabular}

6). Verificou-se, ainda, que o nível de atividade física não está associado à exposição a comportamentos sedentários nem nos dias de semana nem nos finais de semana.

\section{Discussão}

Este estudo apresenta evidências tanto em relação à prevalência quanto em relação aos fatores associados à exposição a níveis insuficientes de prática de atividades físicas e comportamentos sedentários em adolescentes. As prevalências observadas foram altas em relação a esses dois comportamentos.
Um importante resultado deste estudo foi a identificação de que a participação nas aulas de educação física é um fator associado tanto ao nível de prática de atividades físicas quanto à exposição a comportamento sedentário. Além deste fator, os resultados indicam que o local de residência, o turno, a situação ocupacional e o sexo discriminaram significativamente estas condutas de saúde.

Este estudo tem como principais pontos positivos o fato de ter sido conduzido com uma amostra razoavelmente precisa (tamanho amostral) e de ser representativa dos adolescentes estudantes do ensino 
Tabela 2 - Prevalência de insuficientemente ativos em adolescentes segundo variáveis demográficas e socioeconômicas. Table 2 - Prevalence of insufficient activity levels among adolescents by demographic and socioeconomic variables.

\begin{tabular}{|c|c|c|c|c|c|c|c|c|c|c|}
\hline \multirow[t]{2}{*}{ Variável } & & \multicolumn{3}{|c|}{ Rapazes } & \multicolumn{3}{|c|}{ Moças } & \multicolumn{3}{|c|}{ todos } \\
\hline & & $\%$ & $\mathrm{~N}$ & $p$ & $\%$ & $\mathrm{~N}$ & $\mathrm{p}$ & $\%$ & $\mathrm{~N}$ & $\mathrm{p}$ \\
\hline \multirow[t]{2}{*}{ Faixa etária } & 14 a 16 & 59,7 & 357 & & 71,5 & 830 & & 67,5 & 1189 & \\
\hline & 17 a 19 & 56,5 & 614 & 0,20 & 69,0 & 925 & 0,18 & 63,4 & 1543 & 0,00 \\
\hline \multirow[t]{2}{*}{ Turno } & Diurno & 57,5 & 522 & & 69,3 & 1042 & & 64,9 & 1565 & \\
\hline & Noturno & 57,7 & 448 & 0,20 & 71,5 & 711 & 0,18 & 65,5 & 1164 & 0,68 \\
\hline \multirow[t]{3}{*}{ Série } & $1^{\circ}$ ano & 58,0 & 447 & & 70,0 & 764 & & 65,1 & 1214 & \\
\hline & $2^{\circ}$ ano & 62,1 & 324 & 0,00 & 73,2 & 589 & 0,02 & 68,7 & 915 & 0,00 \\
\hline & $3^{\circ}$ ano & 51,2 & 196 & & 66,4 & 395 & & 60,4 & 592 & \\
\hline \multirow[t]{2}{*}{ Local de residência } & Rural & 56,4 & 207 & & 77,6 & 395 & & 69,9 & 606 & \\
\hline & Urbano & 58,0 & 759 & 0,58 & 68,3 & 1348 & 0,00 & 64,1 & 2109 & 0,00 \\
\hline \multirow[t]{2}{*}{ Morar com os pais } & Sim & 56,9 & 642 & & 71,5 & 1075 & & 65,2 & 1721 & \\
\hline & Não & 58,9 & 311 & 0,43 & 68,1 & 666 & 0,06 & 64,8 & 977 & 0,79 \\
\hline \multicolumn{2}{|c|}{ Escolaridade da mãe $>8$ anos } & 58,6 & 636 & & 70,2 & 1237 & & 68,5 & 1879 & \\
\hline & $<8$ anos & 55,4 & 265 & 0,24 & 70,4 & 426 & 0,92 & 63,7 & 691 & 0,22 \\
\hline \multirow[t]{2}{*}{ Cor da pele } & Não-branca & 59,4 & 247 & & 72,1 & 460 & & 67,0 & 707 & \\
\hline & branca & 57,3 & 722 & 0,44 & 69,4 & 1289 & 0,20 & 64,5 & 2017 & 0,14 \\
\hline \multirow[t]{2}{*}{ Trabalho } & Sim & 49,9 & 256 & & 61,9 & 234 & & 55,0 & 492 & \\
\hline & Não & 61,2 & 707 & 0,00 & 71,7 & 1514 & 0,00 & 67,9 & 2224 & 0,00 \\
\hline \multirow[t]{2}{*}{ Educação Física } & Sim & 57,0 & 378 & & 67,4 & 543 & & 62,8 & 924 & \\
\hline & Não & 58,1 & 591 & 0,65 & 71,7 & 1211 & 0,02 & 66,6 & 1805 & 0,01 \\
\hline \multirow[t]{2}{*}{ TV dias de semana } & $<3$ horas dia & 56,1 & 570 & & 70,7 & 1030 & & 64,7 & 1603 & \\
\hline & $\geq 3$ horas dia & 60,0 & 401 & 0,11 & 69,4 & 721 & 0,46 & 65,8 & 1125 & 0,48 \\
\hline \multirow[t]{2}{*}{ TV fim de semana } & $<3$ horas dia & 57,6 & 446 & & 70,6 & 928 & & 65,8 & 1377 & \\
\hline & $\geq 3$ horas dia & 57,7 & 525 & 0,97 & 69,8 & 821 & 0,65 & 64,5 & 1349 & 0,37 \\
\hline
\end{tabular}

médio no Estado de Pernambuco. Além disso, os dados foram coletados mediante a utilização de um questionário que foi previamente testado e apresentou um nível de reprodutibilidade de moderado a alto.

Por outro lado, devido a utilização de questionário e de medidas auto-referidas é importante interpretar com cautela as estimativas de prevalência relatadas neste estudo, principalmente em relação a possível viés de informação. A possibilidade de generalização dos resultados para a população adolescente como um todo é limitada, tendo em vista que o estudo foi conduzido somente com estudantes do ensino médio. Por se tratar de estudo analítico, o delineamento transversal empregado deve ser considerado também como uma limitação devido à possibilidade de causalidade reversa. Por fim, os dados foram coletados em uma região específica do país e, por isso, a generalização dos resultados para outras regiões deve ser efetuada com considerável cuidado.

A maioria dos estudos conduzidos no Brasil foi realizada nas regiões Sul e Sudeste, abrangendo populações específicas e as focalizando isoladamente, na prática de atividades físicas ou, menos frequentemente, na exposição a comportamento sedentário. Até onde se sabe, este é um dos primeiros estudos realizados no Brasil que focalizou simultaneamente a exposição a níveis insuficientes de prática de atividade física e a 
Tabela 3 - Prevalência de exposição a comportamento sedentário em dias de semana segundo variáveis demográficas e socioeconômicas.

Table 3 - Prevalence of sedentary behavior exposure on week days by demographic and socioeconomic variables.

\begin{tabular}{|c|c|c|c|c|c|c|c|c|c|c|c|c|c|}
\hline \multirow{3}{*}{ Variáveis } & & \multicolumn{6}{|c|}{ Comportamento sedentário dias de semana } & \multicolumn{6}{|c|}{ Comportamento sedentário dias de final de semana } \\
\hline & & \multicolumn{2}{|c|}{ Rapazes } & \multicolumn{2}{|c|}{ Moças } & \multicolumn{2}{|c|}{ Todos } & \multicolumn{2}{|c|}{ Rapazes } & \multicolumn{2}{|c|}{ Moças } & \multicolumn{2}{|c|}{ Todos } \\
\hline & & $\%$ & $\mathrm{~N}$ & $\%$ & $\mathrm{~N}$ & $\%$ & $\mathrm{~N}$ & $\%$ & $\mathrm{~N}$ & $\%$ & $\mathrm{~N}$ & $\%$ & $f$ \\
\hline \multirow[t]{2}{*}{ Faixa etária } & 14 a 16 & 47,6 & 280 & 42,9 & 499 & 44,2 & 780 & 59,3 & 355 & 49,9 & 578 & 53,0 & 934 \\
\hline & 17 a 19 & 35,8 & 390 & 40,5 & 543 & 38,5 & 936 & 51,1 & 556 & 44,7 & 600 & 47,6 & 1160 \\
\hline \multicolumn{2}{|c|}{ Valor $p$} & \multicolumn{2}{|c|}{$\left(p \_0,001\right)$} & \multicolumn{2}{|c|}{$(p=0,22)$} & \multicolumn{2}{|c|}{$\left(p \_0,001\right)$} & \multicolumn{2}{|c|}{$\left(p \_0,001\right)$} & \multicolumn{2}{|c|}{$(p=0,01)$} & \multicolumn{2}{|c|}{$\left(p \_0,001\right)$} \\
\hline \multirow[t]{2}{*}{ Turno } & Diurno & 45,8 & 416 & 43,4 & 653 & 44,3 & 1069 & 58,0 & 527 & 50,7 & 762 & 53,4 & 1289 \\
\hline & Noturno & 32,7 & 254 & 39,0 & 388 & 36,3 & 646 & 49,3 & 383 & 41,7 & 415 & 45,1 & 803 \\
\hline \multicolumn{2}{|c|}{ Valor $p$} & \multicolumn{2}{|c|}{$(p=0,45)$} & \multicolumn{2}{|c|}{$(p=0,02)$} & \multicolumn{2}{|c|}{$\left(p \_0,001\right)$} & \multicolumn{2}{|c|}{$\left(\left(p \_0,001\right)\right.$} & \multicolumn{2}{|c|}{$\left(p \_0,001\right)$} & \multicolumn{2}{|c|}{$\left(p \_0,001\right)$} \\
\hline \multirow[t]{3}{*}{ Série } & $1^{\circ}$ ano & 39,2 & 305 & 40,5 & 443 & 39,9 & 748 & 51,6 & 402 & 42,8 & 527 & 49,5 & 923 \\
\hline & $2^{\circ}$ ano & 41,8 & 218 & 43,9 & 359 & 43,2 & 580 & 57,5 & 300 & 46,6 & 380 & 51,0 & 680 \\
\hline & $3^{\circ}$ ano & 37,8 & 146 & 40,5 & 239 & 39,5 & 856 & 54,0 & 208 & 45,8 & 270 & 48,6 & 476 \\
\hline \multicolumn{2}{|c|}{ Valor $\mathrm{p}$} & $(p=$ & & $(p=$ & & $(p=$ & 10) & $(p=$ & & $(p=$ & 05) & & 50) \\
\hline Local de residênc & ia Rural & 31,4 & 115 & 43,9 & 868 & 32,2 & 283 & 48,9 & 179 & 47,3 & 241 & 47,9 & 421 \\
\hline & Urbano & 41,8 & 549 & 32,9 & 359 & 43,1 & 1420 & 55,3 & 725 & 46,9 & 926 & 50,3 & 1655 \\
\hline Valor $\mathrm{p}$ & & (p_c & 01) & (p_ & 01) & (p_c & $01)$ & $(p=$ & & $(p=$ & 86) & & 20) \\
\hline Morar com pais & Sim & 39,5 & 446 & 42,6 & 642 & 41,2 & 1089 & 52,9 & 598 & 48,5 & 730 & 50,4 & 1330 \\
\hline & Não & 40,8 & 216 & 40,0 & 391 & 40,3 & 608 & 56,9 & 301 & 45,0 & 439 & 49,2 & 741 \\
\hline Valor $p$ & & $(p=$ & & $(p=$ & & $(p=$ & 57) & $(p=$ & & $(p=$ & 08) & $(p=$ & 47) \\
\hline Escolaridade da & $\geq 8$ anos & 37,8 & 411 & 40,6 & 715 & 39,6 & 1130 & 53,4 & 580 & 46,7 & 822 & 49,2 & 1406 \\
\hline mãe & $>$ 8anos & 44,7 & 214 & 45,5 & 276 & 45,1 & 490 & 56,4 & 270 & 48,7 & 295 & 52,1 & 566 \\
\hline Valor $\mathrm{p}$ & & $(p=$ & & $(p=$ & & (p_c & $001)$ & $(p=$ & & $(p=$ & 39) & & 10) \\
\hline Cor da pele branc & a Não & 38,7 & 489 & 41,4 & 770 & 42,5 & 447 & 53,8 & 680 & 46,8 & 869 & 49,6 & 1553 \\
\hline & Sim & 42,7 & 178 & 42,4 & 269 & 40,3 & 1263 & 54,3 & 226 & 47,9 & 305 & 50,5 & 532 \\
\hline Valor $p$ & & $(p=$ & & $(p=$ & & $(p=$ & 22) & $(p=$ & & $(p=$ & 63) & $(p=$ & 542) \\
\hline Trabalho & Sim & 25,3 & 130 & 25,9 & 98 & 25,6 & 229 & 51,3 & 263 & 41,4 & 157 & 47,0 & 421 \\
\hline & Não & 46,2 & 535 & 44,6 & 943 & 45,2 & 1480 & 55,3 & 640 & 48,3 & 1019 & 50,8 & 1662 \\
\hline Valor $\mathrm{p}$ & & $\left(p \_c\right.$ & 01) & (p_ & 01) & (p_c & 01) & $(p=$ & & $(p=$ & 01) & $(p=$ & 04) \\
\hline Educação Física & Sim & 37,7 & 251 & 40,5 & 325 & 39,2 & 577 & 54,5 & 362 & 48,5 & 390 & 51,3 & 753 \\
\hline & Não & 40,9 & 416 & 42,1 & 714 & 41,7 & 1133 & 53,7 & 547 & 46,6 & 784 & 49,3 & 1338 \\
\hline Valor $p$ & & $(p=$ & & $(p=$ & & $(p=$ & 12) & $(p=$ & & & 37) & & 24) \\
\hline
\end{tabular}

comportamento sedentário.

Apesar de utilizar instrumento de medida diferente em comparação ao utilizado em outros estudos, os pontos de corte para determinação do desfecho foi o mesmo. No presente estudo, a exposição a níveis insuficientes de atividade física foi superior ao observado em estudantes do ensino médio de Santa Catarina ${ }^{33}$ e em adolescentes de Pelotas ${ }^{23}$. Também foi maior que os resultados encontrados em estudantes do ensino médio de escolas particulares de Recife ${ }^{24}$. Entretanto, observaram-se índices mais elevados, como no estudo com estudantes das redes pública e particular de Maceió ${ }^{25}$, e no estudo realizado com estudantes na cidade de Niterói ${ }^{22}$.

Similarmente ao observado nos estudos sintetizados em revisão sistemática ${ }^{28}$ sobre o assunto, a proporção de moças expostas a níveis insuficientes de prática de atividade física foi significativamente maior do que 
Tabela 4 - Odds Ratio (OR) bruto e ajustado e respectivos intervalos de confiança (IC95\%) para fatores demográficos, socioeconômicos e relacionados à escola, considerando níveis insuficientes de prática de atividade física como desfecho. Table 4 - Crude and adjusted Odds Ratio (OR) and 95\% Confidence Intervals (95\% Cl) for demographic, socioeconomic and school-related variables considering insufficient levels of physical activity as the outcome.

\begin{tabular}{|c|c|c|c|c|c|c|c|}
\hline Nível & Variáveis & $\begin{array}{c}\text { OR } \\
\text { Bruto }\end{array}$ & IC 95\% & Valor $\mathrm{p}$ & OR ajustado & IC 95\% & Valor $\mathrm{p}$ \\
\hline \multirow{9}{*}{1} & Sexo & & & & & & \\
\hline & Rapazes & 1 & & & 1 & & \\
\hline & Moças & 1,73 & $1,52-1,96$ & $<0,01$ & 1,69 & $1,48-1,92$ & 0,00 \\
\hline & Faixa etária & & & & & & \\
\hline & 14 a 16 & 1 & & & 1 & & \\
\hline & 17 a 19 & 0,83 & $0,73-0,95$ & $<0,01$ & 0,88 & $0,78-1,01$ & 0,08 \\
\hline & Cor da pele & & & & & & \\
\hline & Branca & 1 & & & 1 & & \\
\hline & Não-branca & 0,89 & $0,77-1,03$ & 0,14 & 0,89 & $0,76-1,05$ & 0,17 \\
\hline \multirow{12}{*}{2} & Local de residência & & & & & & \\
\hline & Urbano & 1 & & & 1 & & \\
\hline & Rural & 1,23 & $1,05-1,45$ & $<0,01$ & 1,28 & $1,08-1,53$ & 0,00 \\
\hline & Morar com os pais & & & & & & \\
\hline & Sim & 1 & & & 1 & & \\
\hline & Não & 0,98 & $0,86-1,12$ & 0,79 & 0,99 & $0,86-1,14$ & 0,92 \\
\hline & Escolaridade da mãe & & & & & & \\
\hline & $<8$ anos & 1 & & & 1 & & \\
\hline & $\geq 8$ anos & 0,91 & $0,78-1,05$ & 0,22 & 0,99 & $0,87-1,18$ & 0,93 \\
\hline & Trabalho & & & & & & \\
\hline & Não & 1 & & & 1 & & \\
\hline & Sim & 0,57 & $0,49-0,67$ & $<0,01$ & 0,65 & $0,55-0,76$ & 0,00 \\
\hline \multirow{10}{*}{3} & Turno & & & & & & \\
\hline & Diurno & 1 & & & 1 & & \\
\hline & Noturno & 1,02 & $0,85-1,10$ & 0,68 & 1,17 & $1,01-1,37$ & 0,03 \\
\hline & Série & & & & & & \\
\hline & $1^{\circ}$ ano & 1 & & & 1 & & \\
\hline & $2^{\circ}$ ano & 1,20 & $1,36-1,39$ & 0,01 & 0,83 & $0,69-0,99$ & 0,05 \\
\hline & $3^{\circ}$ ano & 0,82 & $0,70-0,97$ & 0,02 & 0,66 & $0,55-0,80$ & 0,00 \\
\hline & Educação Física & & & & & & \\
\hline & Não & 1 & & & 1 & & \\
\hline & Sim & 0,84 & $0,74-0,96$ & $<0,01$ & 0,86 & $0,74-0,99$ & 0,04 \\
\hline
\end{tabular}

entre os rapazes. O odds de relatar níveis insuficientes de prática de atividades físicas foi $69 \%$ superior entre as moças em comparação com os rapazes. Esta evidência coincide com os achados de alguns estudos relatados na literatura ${ }^{23,34,35}$.

Verificou-se que os estudantes residentes em áreas rurais apresentaram uma chance $28 \%$ superior de serem classificados como insuficientemente ativos. Esse resultado pode estar associado à maior oferta de áreas públicas de lazer nos centros urbanos, tais como praças e quadras. Observou-se ainda que os adolescentes que relataram trabalhar apresentaram menor chance de apresentar níveis insuficientes de atividade física, corroborando os resultados de outras investigações ${ }^{23,36-38}$.

Estudos conduzidos com crianças e adolescentes brasileiros identificaram outros fatores associados a níveis insuficientes de atividade física. Em Pelotas, aqueles que 
Tabela 5 - Odds Ratio (OR) bruto e ajustado e respectivos intervalos de confiança (IC95\%) para fatores demográficos, socioeconômicos e relacionados à escola, considerando a exposição a comportamento sedentário em dias de semana como desfecho.

Table 5 - Crude and adjusted Odds Ratio (OR) and 95\% Confidence Intervals (95\% Cl) for demographic, socioeconomic and school-related variables considering the exposure to sedentary behavior on week days as the outcome.

\begin{tabular}{|c|c|c|c|c|c|c|c|}
\hline Nível & Variáveis & $\begin{array}{c}\text { OR } \\
\text { Bruto }\end{array}$ & IC 95\% & Valor $\mathrm{p}$ & OR ajustado & IC $95 \%$ & Valor $\mathrm{p}$ \\
\hline \multirow{9}{*}{1} & Sexo & & & & & & \\
\hline & Rapazes & 1 & & & 1 & & \\
\hline & Moças & 1,08 & $0,95-1,22$ & 0,21 & 1,05 & $0,92-1,19$ & 0,41 \\
\hline & Faixa etária & & & & & & \\
\hline & 14 a 16 & 1 & & & 1 & & \\
\hline & 17 a 19 & 0,78 & 0,69-1,89 & 0,00 & 0,79 & $0,69-0,89$ & 0,00 \\
\hline & Cor da pele & & & & & & \\
\hline & Branca & 1 & & & 1 & & \\
\hline & Não-branca & 0,91 & $0,79-1,05$ & 0,22 & 0,91 & $0,79-1,05$ & 0,20 \\
\hline \multirow{12}{*}{2} & Local de residência & & & & & & \\
\hline & Urbano & 1 & & & 1 & & \\
\hline & Rural & 0,62 & $0,53-0,73$ & 0,00 & 0,65 & $0,55-0,77$ & 0,00 \\
\hline & Morar com os pais & & & & & & \\
\hline & Sim & 1 & & & 1 & & \\
\hline & Não & 0,96 & 0,84-1,09 & 0,57 & 0,92 & $0,80-1,05$ & 0,24 \\
\hline & Escolaridade da mãe & & & & & & \\
\hline & $<8$ anos & 1 & & & 1 & & \\
\hline & $\geq 8$ anos & 1,25 & $1,08-1,44$ & 0,00 & 1,01 & $0,80-1,29$ & 0,93 \\
\hline & Trabalho & & & & & & \\
\hline & Não & 1 & & & 1 & & \\
\hline & Sim & 0,41 & $0,34-0,49$ & 0,00 & 0,42 & $0,34-0,49$ & 0,00 \\
\hline \multirow{10}{*}{3} & Turno & & & & & & \\
\hline & Diurno & 1 & & & 1 & & \\
\hline & Noturno & 0,71 & $0,63-0,81$ & 0,00 & 0,80 & $0,69-0,93$ & 0,00 \\
\hline & Série & & & & & & \\
\hline & $1^{\circ}$ ano & 1 & & & 1 & & \\
\hline & $2^{\circ}$ ano & 1,14 & $0,99-1,32$ & 0,06 & 1,02 & $0,85-1,22$ & 0,81 \\
\hline & $3^{\circ}$ ano & 0,98 & $0,84-1,15$ & 0,83 & 0,92 & $0,76-1,14$ & 0,81 \\
\hline & Educação Física & & & & & & \\
\hline & Não & 1 & & & 1 & & \\
\hline & Sim & 0,90 & $0,79-1,02$ & 0,12 & 0,80 & $0,69-0,92$ & 0,00 \\
\hline
\end{tabular}

relataram elevado nível socioeconômico, ter mãe inativa e maior tempo diário assistindo televisão apresentaram maior chance de ser classificado com baixo nível de atividade física (nomeado pelos autores do estudo como sedentários ${ }^{23}$.

Na Cidade de São Paulo, em estudo com crianças de escolas públicas, foram identificados diversos fatores biológicos e sociodemográficos atribuídos à inatividade física. No referido estudo, observou-se que a inatividade física estava positivamente associada à ocorrência de sobrepeso, ao aumento da idade, a referir ter bom apetite, à coleta de lixo na residência inferior a duas vezes por semana e à mãe trabalhar fora de casa ${ }^{34}$.

A prevalência de exposição a comportamento sedentário foi inferior ao observado entre adolescentes da cidade de 
Tabela 6 - Odds Ratio (OR) bruto e ajustado e respectivos intervalos de confiança (IC95\%) para fatores demográficos, socioeconômicos e relacionados à escola, considerando a exposição a comportamento sedentário em dias de final de semana como desfecho.

Table 6 - Crude and adjusted Odds Ratio (OR) and 95\% Confidence Intervals (95\% Cl) for demographic, socioeconomic and school-related variables considering exposure to sedentary behavior on weekend days as the outcome.

\begin{tabular}{|c|c|c|c|c|c|c|c|}
\hline Nível & Variáveis & $\begin{array}{c}\text { OR } \\
\text { Bruto }\end{array}$ & IC 95\% & Valor $\mathrm{p}$ & OR ajustado & IC 95\% & Valor $\mathrm{p}$ \\
\hline \multirow{9}{*}{1} & Sexo & & & & & & \\
\hline & Rapazes & 1 & & & 1 & & \\
\hline & Moças & 0,75 & $0,67-0,85$ & 0,00 & 0,73 & $0,65-0,83$ & 0,00 \\
\hline & Faixa etária & & & & & & \\
\hline & 14 a 16 & 1 & & & 1 & & \\
\hline & 17 a 19 & 0,80 & $0,71-0,91$ & 0,00 & 0,77 & $0,68-0,87$ & 0,00 \\
\hline & Cor da pele & & & & & & \\
\hline & Branca & 1 & & & 1 & & \\
\hline & Não-branca & 0,96 & $0,84-1,11$ & 0,64 & 0,96 & $0,84-1,11$ & 0,63 \\
\hline \multirow{12}{*}{2} & Local de residência & & & & & & \\
\hline & Urbano & 1 & & & 1 & & \\
\hline & Rural & 0,90 & $0,78-1,05$ & 0,20 & 0,95 & $0,81-1,12$ & 0,57 \\
\hline & Morar com os pais & & & & & & \\
\hline & Sim & 1 & & & 1 & & \\
\hline & Não & 0,95 & $0,84-1,08$ & 0,47 & 0,98 & $0,86-1,13$ & 0,87 \\
\hline & Escolaridade da mãe & & & & & & \\
\hline & $<8$ anos & 1 & & & 1 & & \\
\hline & $\geq 8$ anos & 1,12 & $0,97-1,29$ & 0,10 & 0,95 & $0,73-1,25$ & 0,68 \\
\hline & Trabalho & & & & & & \\
\hline & Não & 1 & & & 1 & & \\
\hline & Sim & 0,85 & $0,74-0,99$ & 0,04 & 0,82 & $0,70-0,96$ & 0,01 \\
\hline \multirow{10}{*}{3} & Turno & & & & & & \\
\hline & Diurno & 1 & & & 1 & & \\
\hline & Noturno & 0,71 & $0,63-0,81$ & 0,00 & 0,71 & $0,61-0,82$ & 0,00 \\
\hline & Série & & & & & & \\
\hline & $1^{\circ}$ ano & 1 & & & 1 & & \\
\hline & $2^{\circ}$ ano & 1,06 & $0,92-1,22$ & 0,40 & 1,04 & $0,87-1,25$ & 0,61 \\
\hline & $3^{\circ}$ ano & 0,98 & $0,84-1,15$ & 0,80 & 0,95 & $0,79-1,13$ & 0,59 \\
\hline & Educação Física & & & & & & \\
\hline & Não & 1 & & & 1 & & \\
\hline & Sim & 1,07 & $0,94-1,22$ & 0,24 & 0,98 & $0,85-1,12$ & 0,78 \\
\hline
\end{tabular}

Florianópolis ${ }^{39}$. Além disso, diferentemente do encontrado entre os adolescentes de Florianópolis ${ }^{40}$, no presente estudo a proporção de sujeitos expostos a comportamento sedentário (tempo de assistência à televisão) nos finais de semana foi significativamente maior entre os rapazes do que entre as moças. Verificou-se, ainda, que os estudantes mais velhos (17 a 19 anos) e aqueles que estudam no período da noite apresentaram menor chance de exposição a comportamento sedentário.

Como mencionado, no presente estudo não se observou associação significativa entre o nível de prática de atividades físicas e a exposição a comportamento sedentário. Entretanto, há estudos em que esta independência foi observada. Por exemplo, resultados de estudo desenvolvido com estudantes de Santa Catarina demonstra- 
ram que a chance de ser classificado como "pouco ativo" foi maior entre os rapazes que assistiam duas ou mais horas por dia de televisão ${ }^{40}$. Em adolescentes americanos, evidenciou-se que a prática de atividades físicas estava associada ao tempo gasto assistindo televisão, mas somente entre as moças ${ }^{41}$. Similarmente, estudo com adolescentes canadenses demonstrou que aqueles que despendiam maior tempo assistindo televisão também eram mais inativos fisicamente ${ }^{42}$.

Os resultados deste estudo permitem concluir que a prevalência de exposição a nível insuficiente de prática de atividades fí- sicas é alta, particularmente entre as moças. Os rapazes, por sua vez parecem estar mais expostos a comportamento sedentário. É importante ressaltar que a prática regular de atividade física independe de outros comportamentos do dia a dia que exijam menos esforço, como por exemplo: assistir televisão, ler ou conversar com os amigos. A participação nas aulas de educação física foi um fator associado tanto à prática de atividades físicas quanto à exposição a comportamento sedentário em dias do final de semana e esta evidência deverá ser investigada com maior profundidade em futuros estudos.

\section{Referência}

1. Pate R, Pratt M, Blair SN, Haskell WL, Macera CA, Bouchard C. Physical activity and public health: a recommendation from the Centers for Disease Control and Prevention and the American College of Sports Medicine. JAMA 1995; 273: 402-7.

2. US Department of Health and Human Services. Physical Activity and Health: A Report of the Surgeon General. Atlanta, GA: Centers for Disease Control and Prevention, National Center for Chronic Disease Prevention and Health Promotion, The President's Council on Physical Fitness and Sports; 1996.

3. Slattery ML, Edwards SL, Boucher KM, Anderson K, Caan BJ. Lifestyle and colon cancer: an assessment of factors associated with risk. Am J Epidemiol 1999; 150: 869-77.

4. Srivastava A, Kreiger N. Relation of physical activity to risk of testicular cancer. Am J Epidemiol 2000; 151: 78-87.

5. Hu G, Barengo NC, Tuomilehto J, Lakka TA, Nissinen A, Jousilahti P. Relationship of physical activity and body mass index to the risk of hypertension: a prospective study in Finland. Hypertension 2004; 43: 25-30.

6. Padilla J, Wallace JP, Parks S. Accumulation of physical activity reduces blood pressure in pre- and hypertension. Med Sci Sports Exerc 2005; 37: 1264-75.

7. Rippe JM, Hess S. The role of physical activity in prevention and management of obesity. J Am Diet Assoc 1998; 98(S2): 31-8.

8. Jakicic JM, Otto AD. Physical activity considerations for the treatment and prevention of obesity. Am J Clin Nutr 2005; 82(Suppl 1):226-29.

9. Sallis JF, Patrick K. Physical activity guidelines for adolescents: consensus statement. Pediatr Exerc Sci, Champaign 1994; 6.
10. Erlichman J, Kerbev AL, James WP. Physical activity and its impact on health outcomes. Paper 2: Prevention of unhealthy weight gain and obesity by physical activity: an analysis of the evidence. Obes Rev 2002; 3: 273-87.

11. Maia JA, Lefevre J, Claessens A, Renson R, Vanreusel B, Beunen G. Tracking of physical fitness during adolescence: a panel study in boys. Med Sci Sports Exerc 2001; 33: 765-71.

12. Hardin DS, Hebert JD, Bayden T, Dehart M, Mazur L. Treatment of childhood syndrome X. Pediatrics 1997; 100: E5.

13. Owens S, Gutin B, Allison J, Riggs S, Ferguson M, Litaker $\mathrm{M}$, et al. Effect of physical training on total and visceral fat in obese children. Med Sci Sports Exerc 1999; 31: 143-8.

14. Gutin B, Barbeau P, Owens S, Lemmon CR, Bauman $\mathrm{M}$, Allison J, et al. Effects of exercise intensity on cardiovascular fitness, total body composition, and visceral adiposity of obese adolescents. Am J Clin Nutr 2002; 75: 818-26.

15. Hallal PC, Victora CG, Azevedo MR, Wells JC. Adolescent physical activity and health: a systematic review. Sports Med 2006; 36: 1019-30.

16. Azevedo MR, Araújo CL, Silva MC, Hallal PC. Tracking of physical activity from adolescence to adulthood: a population-based study. Rev Saúde Pública 2007; 41(1): 69-75

17. Sallis, J. F; Patrick, K. J. \& Long, B. J. Overview of the international consensus conference on physical activity guidelines for adolescents. Pediatr Exerc Sci 1994; 6: 299301 .

18. Sallis, J. F. \& Patrick, K. Physical Activity Guidelines for Adolescents: Consensus Statement. Pediatr Exerc Sci 1994; 6(2): 302-4. 
19. Bidlle S, Sallis, JF, Cavill NA. Young and active? Young people and health enhancing physical activity: Evidence and implications. London: Health Education Authority; 1998.

20. Cavil N, Bidlle S, Sallis J. Health enhancing physical activity for young people: statement of United Kingdom expert consensus conference. Pediatr Exerc Sci 2001; 13: $12-25$.

21. Oehlschlaeger MHK, Pinheiro RT, Horta B, Gelatti C, San'Tana P. Prevalência e fatores associados ao sedentarismo em adolescentes de área urbana. Rev Saúde Pública 2004; 38: 157-63.

22. Silva MAM, Rivera IR, Ferraz MRMT, Pinheiro AJT, Alves SWS, Moura AA, et al. Prevalência de fatores de risco cardiovascular em crianças e adolescentes da rede de ensino da cidade de Maceió. Arq Bras Cardiol 2005; 84: 387-92.

23. Hallal PC, Bertoldi AC, Gonçalves H, Victora CG. Prevalence of sedentary lifestyle and associated factors in adolescents 10 to 12 years of age. Cad Saúde Pública 2006: 22: 1277-87.

24. Souza GS, Duarte MFS. Estágios de mudança de comportamento relacionados à atividade física em adolescentes. Rev Bras Med Esporte 2005; 11: 104-8.

25. Silva MAM, Rivera IR, Ferraz MRMT, et al. Prevalência de fatores de risco cardiovascular em crianças e adolescentes da rede de ensino da cidade de Maceió. Arq Bras Cardiol 2005, 84: 5.

26. Tassitano, RM, Bezerra J, Tenório MCM, Colares V, Barros MVG, Hallal PC. Atividade física em adolescentes brasileiros: uma revisão sistemática. Rev Bras Cineantropom Desempenho Hum 2007; 9: 60-5.

27. Robinson TN. Reducing children's television viewing to prevent obesity: a randomized controlled trial. J Am Med Assoc 1999; 282: 1561-7.

28. Tudor-Locke C, Meyers AM. Challenges and opportunities for measuring physical activity in sedentary adults. Sports Med 2001; 31: 91-100

29. Livingstone MBE, Robson PJ, Wallace JMW, McKinley MC. How active are we? Levels of routine physical activity in children and adults. Proc Nutr Soc 2003; 62: 681-701.

30. Biddle SJH, Gorely T, Marshall SJ, Murdey I, Cameron N. Physical activity and sedentary behaviors in youth: issues and controversies. J R Soc Health 2004; 124(1): 2933

31. Rosenberg DE, Bull FC, Marshall AL, Sallis JF, Bauman AE. Assessment of Sedentary Behavior With the International Physical Activity Questionnaire. J Phys Act Health 2008; 5(S1): 30-44.

32. Victora, CG, Huttly SR, Fuchs SC, Olinto MT. The role of conceptual frameworks in epidemiological analysis hierarchical approach. Int J Epidemiol. 1997; 26(1): 2247 .
33. Barros MVG. Atividades físicas e padrão de consumo alimentar em estudantes do ensino médio em Santa Catarina [tese de doutorado]. Universidade Federal do Rio Grande do Sul; 2004.

34. Bracco, Mario M. et al. Modelo hierárquico multivariado da inatividade física em crianças de escolas públicas. $J$ Pediatr 2006; 82: 4.

35. Jenovesi Jf, Bracco MM, Colugnati FA, Taddei JA. Perfil de atividade física em escolares da rede pública de diferentes estados nutricionais. Rev Bras Ci Mov 2003; 11: 4 .

36. Guedes DP, Guedes JERP, Barbosa DS, Oliveira JA. Níveis de prática de atividade física habitual em adolescentes. Rev Bras Med Esporte 2001; 7: 187-200.

37. Shi Z, Lein N, Kumar BN, Holmboe-Ottesen G. Physical activity and associated socio-demographic factors among school adolescents in Jiangsu Province, China. Prev Med 2006; 43: 218-21.

38. Farias Junior J C. Associação entre Prevalência de Inatividade Física e Indicadores de Condição Socioeconômica em Adolescentes. Rev Bras Med Esporte 2008; $14(2)$.

39. Pelegrini A, SilvaRCR, Petroski. Relação entre o tempo em frente à tv e o gasto calórico em adolescentes com diferentes percentuais de gordura corporal Rev Bras Cineantropom. Desempenho Hum 2008;10(1): 81-4.

40. Silva, Kelly Samara da et al. Associações entre atividade física, índice de massa corporal e comportamentos sedentários em adolescentes. Rev Bras Epidemiol [online] 2008; 11(1); 159-68. [Acessado em 28 de outubro de 2008]

41. Lowry R., Wechsler H, Galuska DA, Fulton JE, Kann L. Television Viewing and its Associations with Overweight, Sedentary Lifestyle, and Insufficient Consumption of Fruits and Vegetables Among US High School Students: Differences by Race, Ethnicity, and Gender. J Sch Health 2002; 72(10): 413-21.

42. Koezuka N, Koo M, Allison KR, Adlaf EM, Dwyer JJM, Faulkner G et al. The Relationship between Sedentary Activities and Physical Inactivity among adolescents: Results from the Canadian Community Health Survey. $J$ Adolesc Health 2006; 39: 515-22.

43. Kelder SH, Perry CL, Klepp KI, Lytle LL. Longitudinal tracking of adolescent smoking, physical activity, and food choice behaviors. Am J Public Health 1994; 84: 1121-6.

Recebido em: 26/02/09

Versão final reapresentada em: 19/10/09 Aprovado em: 30/11/09 